\title{
Bifurcations of a creeping air-water flow in a conical container
}

Balci, Adnan; Brøns, Morten; Herrada, Miguel A.; Shtern, Vladimir N.

Published in:

Theoretical and Computational Fluid Dynamics

Link to article, DOI:

$10.1007 / \mathrm{s} 00162-016-0391-z$

Publication date:

2016

Document Version

Peer reviewed version

Link back to DTU Orbit

Citation (APA):

Balci, A., Brøns, M., Herrada, M. A., \& Shtern, V. N. (2016). Bifurcations of a creeping air-water flow in a conical container. Theoretical and Computational Fluid Dynamics, 30(5), 485-496. https://doi.org/10.1007/s00162-016$0391-z$

\section{General rights}

Copyright and moral rights for the publications made accessible in the public portal are retained by the authors and/or other copyright owners and it is a condition of accessing publications that users recognise and abide by the legal requirements associated with these rights.

- Users may download and print one copy of any publication from the public portal for the purpose of private study or research.

- You may not further distribute the material or use it for any profit-making activity or commercial gain

- You may freely distribute the URL identifying the publication in the public portal

If you believe that this document breaches copyright please contact us providing details, and we will remove access to the work immediately and investigate your claim. 


\title{
Bifurcations of a creeping air-water flow in a conical container
}

\author{
Adnan Balci ${ }^{1}$, Morten Brøns ${ }^{1}$, Miguel A. Herrada ${ }^{2}$ and Vladimir N. Shtern ${ }^{3}$ \\ ${ }^{1}$ DTU Compute, Technical University of Denmark 2800 Kgs. Lyngby, Denmark \\ ${ }^{2}$ E.S.I, Universidad de Sevilla, Camino de los Descubrimientos s/n 41092, Spain \\ ${ }^{3}$ Shtern Research and Consulting, Houston, Texas 77096, USA
}

This numerical study describes the eddy emergence and transformations in a slow steady axisymmetric air-water flow, driven by a rotating top disk in a vertical conical container. As water height $H_{\mathrm{w}}$ and cone half-angle $\beta$ vary, numerous flow metamorphoses occur. They are investigated for $\beta=30^{\circ}, 45^{\circ}$ and $60^{\circ}$. For small $H_{\mathrm{w}}$, the air flow is multi-cellular with clockwise meridional circulation near the disk. The air flow becomes one-cellular as $H_{\mathrm{w}}$ exceeds a threshold depending on $\beta$. For all $\beta$, the water flow has an unbounded number of eddies whose size and strength diminish as the cone apex is approached. As the water level becomes close to the disk, the outmost water eddy with clockwise meridional circulation expands, reaches the interface, and induces a thin layer with anticlockwise circulation in the air. Then this layer expands and occupies the entire air domain. The physical reasons for the flow transformations are provided. The results are of fundamental interest and can be relevant for aerial bioreactors.

PACS numbers: 47.32.C-, 47.15-x, 47.55. $-\mathrm{t}$

\section{Introduction}

The emergence of a local circulation region in a swirling flow, often referred to as vortex breakdown (VB), plays an important role in nature (tornadoes) and technology (delta-wing aircraft, combustion and vortex reactors) [1]. It was recently revealed that VB occurs even in a creeping flow $[2,3]$ that can be important for bioreactors.

Bioreactors are a rapidly developing technology. They employ an air-water flow for the efficient growth of tissue culture. A rotating disk [4] or a propeller [5], located in the air region, induces a swirling motion. The swirling air converges toward the reactor axis near the interface and drives the meridional circulation and rotation of water. The air meridional circulation delivers oxygen to the interface required for the tissue culture growth. The water circulation enhances mixing of the dissolved oxygen with other ingredients. The tissue growth is a time- 
consuming process requiring a slow motion of ingredients and small shear stresses. The air driving satisfies these requirements.

Commercial bioreactors are cylindrical. The pattern of an air-water flow depends there on the water height $H_{\mathrm{w}}$ [2]. For small $H_{\mathrm{w}}$ in a cylindrical container with a rotating top disk, the air meridional circulation is clockwise and the water meridional circulation is anticlockwise. As $H_{\mathrm{w}}$ increases, clockwise circulation emerges near the bottom center. This transformation occurs via the swirl decay mechanism similar to that which causes the VB development in a high-speed swirling flow [6].

In a swirling flow, the centrifugal force typically induces a radial gradient of pressure $p$ : $\partial p / \partial r=\rho v^{2} / r$, where $\mathrm{v}$ is the swirl velocity, $r$ is the distance from the axis, and $\rho$ is the fluid density. As a swirling flow meets a normal wall where $v=0$, the centrifugal force has a secondorder zero, and $\partial p / \partial r$, being unbalanced by the centrifugal force, induces a secondary flow which converges to the rotation axis near the wall and goes away from the wall near the axis [7]. This mechanism, explaining the emergence of the water clockwise circulation near the bottom of cylindrical and truncated conical containers [2,3], does not work near the apex of a conical container. One goal of this paper is to investigate what mechanism works in a cone.

Studies of a flow in a cone have long history. Ackerberg [8] analyzed a sink flow in a cone. Conical sidewalls are beneficial for vortex devices. Examples are hydrocyclones [9, 10] and vortex tubes [11] which have large length-to-radius ratios. Swirl decays downstream due to the wall friction. The conical geometry partially compensates the decay and thus helps sustain the centrifugal force to be sufficiently strong as required for particle and thermal separations. The effect is achieved due to decreasing radial extent in a conical part of device and the angular momentum, $r \mathrm{v}$, which is nearly conserved in a fast flow.

The conical geometry also can be beneficial for aerial bioreactors where the flow is typically slow. The swirl rapidly decays from the top toward the bottom of an aerial bioreactor due to friction at its walls and viscous dissipation dominates in a slow motion. The conical geometry can reduce the swirl decay. This potentially beneficial effect is a practical motivation for our study.

The important difference is that the bottom is planar in a cylindrical container, while a nontruncated conical container has a sharp tip. Even a slow (“creeping”) motion has an unbounded set of eddies near the tip. Such sets were first discovered in a creeping flow between two 
inclined walls [12] and then in a cone, where axisymmetric [13-15] and three-dimensional [1617] eddies were found.

Wakiya [13] revealed that the eddies exists in a cone whose half-angle $\beta$ (i.e., the angle between the axis and the sidewall) is less than $80.9^{\circ}$. This feature of conical flows is important for our study, where $\beta=30^{\circ}, 45^{\circ}$ and $60^{\circ}$. It makes the bifurcation scenario very different from that in the cylindrical container [2] and in a truncated cone [3]. In particular, the difference is in the VB occurrence and particularly in the development of a local counter-circulation in water.

As $H_{\mathrm{w}}$ increases in the cylindrical device, VB first emerges in the water flow near the axisbottom intersection and then in the air flow. In contrast, no VB in the water flow occurs in the conical container. There is no necessity for the counter-circulation to emerge because many such eddies exist in the water at any $H_{\mathrm{w}}$. Instead of the VB emergence, the closest-to-interface eddy, having clockwise circulation, expands upward as $H_{\mathrm{w}}$ increases.

The VB emergence in the air flow is similar for both cylindrical and conical containers: as the water VB cell reaches the interface, it reverses the adjacent air flow and develops a thin layer of the anticlockwise circulation of the air.

The bifurcation variety is enriched due to interactions of the VB cells with the air and water eddies located near the interface-sidewall intersection [18]. The bifurcation scenario, found for the creeping flow, remains unchanged as the Reynolds number $R e$ increases up to a few thousands. The cell multiplicity is beneficial for bioreactors because eddies enhance the ingredient mixing thus making the process of culture growth more uniform. It is important that the shear stresses do not harm the tissue because the flow is slow.

The exploration of topological transformations and VB development in two-fluid flows was initiated by the pioneer paper of Brady et al. [19]. This paper contributes to a new field which can be referred to as "Bifurcating multi-fluid flows" which in turn can be viewed as a part of the topological fluid mechanics whose origin can be traced back to the paper by Arnold [20]. The foundations of topological fluid dynamics are discussed in the book written by Arnold \& Khesin [21] and in the proceedings of two IUTAM Symposia [22, 23]. Our paper is a contribution to the part "Bifurcating creeping multi-fluid flows" of this research field.

In the rest of this paper, we formulate the problem in Sect. 2, describe our numerical technique in Sect. 3, investigate the bifurcation scenario in the cone with $\beta=30^{\circ}$ (Sect. 4 ), $45^{\circ}$ 
(Sect. 5) and $60^{\circ}$ (Sect. 6), explore the effect of finite $R e$ (Sect. 7) and summarize the results in Sect. 8 .

\section{Problem formulation}

\subsection{Flow geometry}

Figure 1 is a schematic of the problem. The lower part, $0<\mathrm{z}<h_{\mathrm{w}}$, of the conical container is filled with water, the upper part, $h_{\mathrm{w}}<z<h$, is filled with air; $h$ is the cone height, which serves as a length scale; $\beta$ is the angle between the axis and the sidewall; $\mathbf{g}$ is the gravitational acceleration. The interface is depicted by the thin horizontal line, $z=h_{\mathrm{w}}$. The top disk of radius $R=h \tan \beta$ is located at $z=h$ and rotates with angular velocity $\omega$. The dimensionless control parameters are $\beta$, the relative water height $H_{\mathrm{w}}=h_{\mathrm{w}} / h$, and the Reynolds number, $R e=\omega h^{2} / \nu_{\mathrm{w}}$, which characterizes the rotation strength; $v_{\mathrm{w}}$ is the kinematic viscosity of water. The motion is creeping if $R e<<1$.

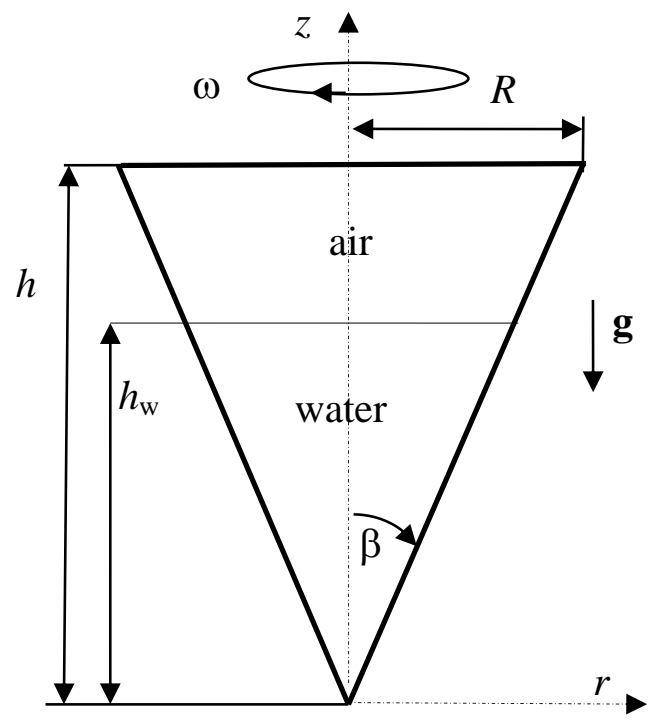

FIG. 1. Schematic of the problem.

Our goal is to study the flow transformations as $H_{\mathrm{w}}$ varies and to find $H_{\mathrm{w}}=H_{\mathrm{wa}}$, at which vortex breakdown (VB) in the air flow develops, depending on $\beta$. 


\subsection{Governing equations}

Using $h, \omega h$, and $\rho_{\mathrm{w}} \omega^{2} h^{2}$ as scales for length, velocity, and pressure, respectively, renders all variables dimensionless. We consider steady axisymmetric flows of air and water both governed by the Navier-Stokes equations for a viscous incompressible fluid,

$$
\begin{aligned}
& r^{-1} \partial(r u) / \partial r+\partial w / \partial z=0 \\
& u \partial u / \partial r+w \partial u / \partial z=v^{2} / r-\rho_{\mathrm{n}} \partial p / \partial r+v_{\mathrm{n}} R^{-1}\left(\nabla^{2} u-u / r^{2}\right) \\
& u \partial \mathrm{v} / \partial r+w \partial \mathrm{v} / \partial z+u \mathrm{v} / r=v_{\mathrm{n}} \operatorname{Re}^{-1}\left(\nabla^{2} \mathrm{v}-\mathrm{v} / r^{2}\right) \\
& u \partial w / \partial r+w \partial w / \partial z=-\rho_{\mathrm{n}} \partial p / \partial z+v_{\mathrm{n}} \operatorname{Re}^{-1}\left(\nabla^{2} w\right)
\end{aligned}
$$

where $\nabla^{2} \equiv r^{-1} \partial(r \partial / \partial r) / \partial r+\partial^{2} / \partial z^{2},(u, v, w)$ are the velocity components in cylindrical coordinates $(r, \phi, z)$, and $p$ is pressure reduced by the hydrostatic contribution. The coefficients, $\rho_{\mathrm{n}}$ and $v_{\mathrm{n}}$, both equal to1 for $\mathrm{n}=1$ (in water) while $\rho_{\mathrm{n}}=\rho_{\mathrm{w}} / \rho_{\mathrm{a}}$ and $v_{\mathrm{n}}=v_{\mathrm{a}} / \nu_{\mathrm{w}}$ for $\mathrm{n}=2$ (in air); $v_{\mathrm{w}}=10^{-6}$

$\mathrm{m}^{2} / \mathrm{s}$ and $v_{\mathrm{a}}=15 \times 10^{-6} \mathrm{~m}^{2} / \mathrm{s}$ are the kinematic viscosities; $\rho_{\mathrm{w}}=1000 \mathrm{~kg} / \mathrm{m}^{3}$ and $\rho_{\mathrm{a}}=1.22 \mathrm{~kg} / \mathrm{m}^{3}$ are the densities; subscripts "a" and "w" are abbreviations for "air" and "water". This air density value corresponds to atmospheric pressure and room temperature.

\subsection{Boundary conditions}

Equations (1)-(4) are solved under the following boundary conditions:

(i) Regularity at the axis, $0<z<1, r=0: u=\mathrm{v}=0, \partial w / \partial r=0$,

(ii) No-slip at the sidewall: $u=\mathrm{v}=w=0$ at $0<z<1, r=z \tan \beta$,

(iii) No-slip, $u=w=0, \mathrm{v}=r$ at the rotating disk, $0<r<\tan \beta, z=1$.

(iii) Continuity of all the velocity and stress components at the air-water interface, $z=F(r)$. In particular, the balance for the normal stresses yields that

$$
p_{\mathrm{w}}-p_{\mathrm{a}}=W e^{-1} \nabla \cdot \mathbf{n}-R e^{-1} \mathbf{n} \cdot\left(\tau_{\mathrm{w}}-\mu_{\mathrm{r}} \tau_{\mathrm{a}}\right) \cdot \mathbf{n}-F r^{-1}\left(1-\rho_{\mathrm{a}} / \rho_{\mathrm{w}}\right) z+C
$$

where $W e=\rho_{\mathrm{w}} \omega^{2} h^{3} / \sigma$ is the Weber number, characterizing the effect of surface tension $\sigma$ at the interface; $F r=\omega^{2} h / g$ is the Froude number, $g=9.81 \mathrm{~m}^{2} / \mathrm{s}$ being the gravitational acceleration magnitude (Fig. 1); $\mathbf{n}$ is the unit vector normal to the interface; $\tau_{\mathrm{w}}$ and $\tau_{\mathrm{a}}$ are tensors of the viscous stresses in water and air, respectively; $\mu_{\mathrm{r}}$ is the air-to-water ratio of the dynamic 
viscosities; $\mathrm{C}$ is a constant which is determined by imposing the mass conservation of the liquid inside the container as the interface is deformed,

$$
6 \int^{a}[F(r)-F(a)] r d r+F(a) a^{2}=H_{w} r_{\mathrm{w}}^{2}
$$

where $r_{\mathrm{w}}=H_{w} \tan \beta$ and $a$ is the radial coordinate where the interface meets the sidewall, and a root of the equation $a=\mathrm{F}(a) \tan \beta$.

\subsection{Reduced problem}

As $R e \rightarrow 0$, the motion becomes very slow and the interface deformation becomes negligible, $F(r) \equiv H_{\mathrm{w}}$. The nonlinear terms become negligibly small compared with the linear terms in equation (3), which reduces to

$$
\nabla^{2} \mathrm{v}-\mathrm{v} / r^{2}=0
$$

This equation has a nonzero solution due to the boundary condition at the disk: $v=r$ at $z=1$. At all other walls, the swirl velocity is zero, $v=0$. At the interface, $v$ and the swirl shear stresses are continuous: $\mathrm{v}_{\mathrm{w}}=\mathrm{v}_{\mathrm{a}}$ and $\partial \mathrm{v}_{\mathrm{w}} / \partial z=\mu_{\mathrm{r}} \partial \mathrm{v}_{\mathrm{a}} / \partial z$ at $z=H_{\mathrm{w}}$. Thus the problem for the swirl velocity becomes separated from the problem for the meridional motion in the limiting case as $R e \rightarrow 0$, similar to that in the one-fluid problem studied by Hills [24]. We first solve this linear problem for swirl.

Next, we address the problem for the meridional motion. Since the boundary conditions are uniform, the meridional motion is only driven by the centrifugal force corresponding to term $\mathrm{v}^{2} / \mathrm{r}$ in equation (2). This term must be preserved, while the other nonlinear terms can be omitted in the limiting case as $R e \rightarrow 0$. Then introducing $u^{*}=u / \operatorname{Re}$ and $w^{*}=w / R e$ reduces equations (1), (2), and (4) to

$$
\begin{aligned}
& r^{-1} \partial\left(r u^{*}\right) / \partial r+\partial w^{*} / \partial z=0, \\
& \rho_{\mathrm{n}} \partial p / \partial r-v^{2} / r=v_{\mathrm{n}}\left(\nabla^{2} u^{*}-u^{*} / r^{2}\right), \\
& \rho_{\mathrm{n}} \partial p / \partial z=v_{\mathrm{n}} \nabla^{2} w^{*} .
\end{aligned}
$$

The velocity and shear-stress components are continuous across the undisturbed interface, $z=$ $H_{\mathrm{w}}$, where the normal-to-interface velocity, $w^{*}$, is zero. It is interesting that the entire problem formally is nonlinear despite the motion is creeping, but can be divided into the two linear 
problems: one for the swirl velocity (7) and the other for the meridional motion (8)-(10). After solving problem (7), the "source" term, $v^{2} / r$ in equation (9), is prescribed, so the problem for the meridional motion also is linear. This feature is also similar to that in the one-fluid flow studied by Hills [14].

\section{Numerical procedures}

\subsection{Transformation of equations}

The numerical technique is similar to that used in Refs. [2], [3] and [25]. We reiterate here the main features for convenience of the reader. For the $R e$ ranges addressed in this work, the interface deformation is negligibly small and $z=z_{\mathrm{i}}=H_{\mathrm{w}}$ is a good approximation for the interface. The number of variables involved in the problem is reduced by introducing a streamfunction-vorticity-angular-momentum form. System (1)-(4) is transformed into three equations for the Stokes stream function $\Psi, u=-r^{-1} \partial \Psi / \partial z, w=r^{-1} \partial \Psi / \partial r$, the azimuthal vorticity component, $\chi=\partial u / \partial z-\partial w / \partial r$, and circulation, $\Gamma=r \mathrm{v}$ :

$$
\begin{aligned}
& \nabla^{2} \Psi-2 r^{-1} \partial \Psi / \partial r=-r \chi \\
& u \partial \chi / \partial r+w \partial \chi / \partial z-u \chi / r=2 r^{-3} \Gamma \partial \Gamma / \partial z+v_{\mathrm{n}} R^{-1}\left(\nabla^{2} \chi-\chi / r^{2}\right), \\
& u \partial \Gamma / \partial r+w \partial \Gamma / \partial z=v_{\mathrm{n}} R^{-1}\left(\nabla^{2} \Gamma-2 r^{-1} \partial \Gamma / \partial r\right),
\end{aligned}
$$

These equations are solved applying the boundary conditions for $\Psi, \eta$ and $\Gamma$ which follows

from those listed in $\$ 2.3$ by substitution $u=-r^{-1} \partial \Psi / \partial z$ and $w=r^{-1} \partial \Psi / \partial r$. Contours $\Psi=$ constant, are used to depict patterns of the meridional motion.

\subsection{Linear problem}

In the limiting case as $R e \rightarrow 0$, introducing $\chi^{*}=\chi / R e$ and $\Psi^{*}=\Psi / \operatorname{Re}$ reduces equations (11)(13) to

$$
\begin{aligned}
& \nabla^{2} \Psi^{*}-2 r^{-1} \partial \Psi * / \partial r=-r \chi^{*}, \\
& 2 r^{-3} \Gamma \partial \Gamma / \partial z+v_{\mathrm{n}}\left(\nabla^{2} \chi^{*}-\chi^{*} / r^{2}\right)=0, \\
& \nabla^{2} \Gamma-2 r^{-1} \partial \Gamma / \partial r=0 .
\end{aligned}
$$




\subsection{Discretization}

A boundary-fitted coordinate system is used to calculate the problem. We use $\eta=\phi / \beta, \phi=$ atan $(r / z)$, in order to have the unit interval $0<\eta<1$ for any $\beta$. Both the water and air regions are mapped onto the fixed rectangular domains (a) $0 \leq \eta \leq 1,0 \leq \xi_{\mathrm{w}} \leq H_{\mathrm{w}}$, and (b) $0 \leq \eta \leq 1, H_{\mathrm{w}} \leq \xi_{\mathrm{a}}$ $\leq 1-H_{\mathrm{w}}$. To this end, we perform the coordinate transformations: (a) $\eta=r /(1-z \tan \beta), \xi_{\mathrm{w}}=z$ and (b) $\eta=r /(1-z \tan \beta), \xi_{\mathrm{a}}=z$. These domains are discretized by using a set of $n_{\xi \mathrm{w}}$ and $\mathrm{n}_{\xi \mathrm{a}}$ Chebychev spectral collocation points in the $\xi$ direction. The $\eta$ interval is discretized using a set of $\mathrm{n}_{\eta}$ Chebychev spectral collocation points.

\subsection{Nonlinear problem}

The Newton iterative procedure is used to solve the discretized non-linear problem derived from (11)-(13) and their corresponding boundary conditions. Given an initial solution guess, a new approximate solution is found by solving the system of $3\left(\mathrm{n}_{\xi \mathrm{w}}+\mathrm{n}_{\xi \mathrm{g}}\right) \times \mathrm{n}_{\varphi}$ linear algebraic equations for $\Psi, \eta$ and $\Gamma$ at the collocation points resulting from the Newton linearization. For a prescribed geometrical configuration, the simulation is started with a small value of the Reynolds number and the flow at rest. Once the approximate solution has converged, the converged solution is used as a new guess for a new run with a higher Reynolds number.

\subsection{Advantages of Chebyshev grid}

In the reduced problem, the discretized problem consists of (i) system of $\left(\mathrm{n}_{\xi \mathrm{w}}+\mathrm{n}_{\xi_{a}}\right) \times \mathrm{n}_{\varphi}$ linear algebraic equations for $\Gamma$ and (ii) system of $2\left(n_{\xi \mathrm{w}}+n_{\xi a}\right) \times n_{\varphi}$ linear algebraic equations for $\Psi^{*}$ and $\eta^{*}$ which are solved sequentially. All linear systems, in the full and reduced problems, are solved directly applying the standard procedure of matrix inversion.

For the presented results, the simulations are done mostly with $\mathrm{n}_{\xi \mathrm{w}}=40, \mathrm{n}_{\xi \mathrm{a}}=30$, and $\mathrm{n}_{\varphi}=50$ (standard grid). In order to verify the grid independence, some runs have been carried out at $\mathrm{n}_{\xi \mathrm{w}}$ $=50, \mathrm{n}_{\xi} \mathrm{a}=40$, and $\mathrm{n}_{\varphi}=60$ (fine grid).

Since the Chebyshev grid points concentrate near the interface from both sides, the approach is adequate to resolve thin circulation layers, located near the interface, even using moderate values of $n_{\xi \mathrm{w}}$ and $\mathrm{n}_{\xi \mathrm{a}}$. The Chebyshev grid points concentrate near the walls as wellwhich helps 
to better resolve the Moffatt and other corner eddies, even using a moderate value of $n_{\varphi}$. The Chebyshev grid points also concentrate near the axis which helps resolve small vortex breakdown bubbles emerging near the axis-bottom and axis-interface intersections as $H_{\mathrm{w}}$ increases.

\section{Bifurcations at $\beta=30^{\circ}$}

First, we explore the bifurcation scenario as the water height, $H_{\mathrm{w}}$, increases in a conical container with $\beta=30^{\circ}$. Fig. 2(a) depicts the pattern of the meridional motion at $H_{\mathrm{w}}=0.1$. The rotating lid, located at $z=1$, induces the centrifugal force which pushes air to the periphery near the top and thus induces the clockwise circulation, indicated by the arrow in Fig. 2(a).

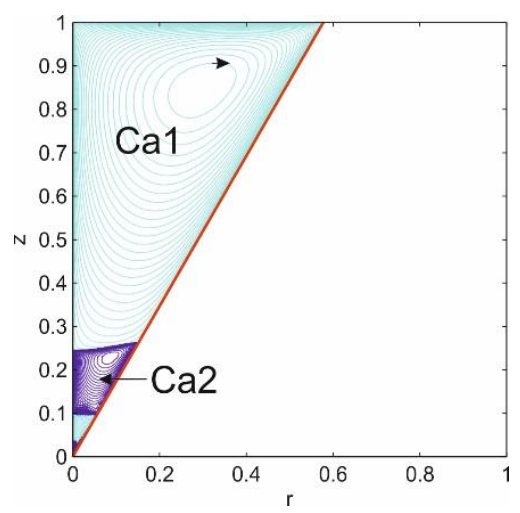

(a) $H_{\mathrm{w}}=0.1$

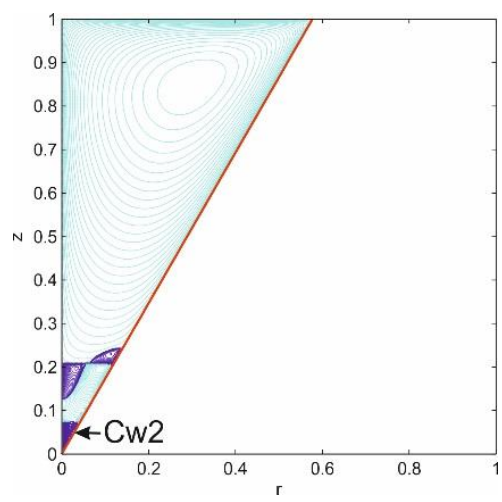

(d) $H_{\mathrm{w}}=0.207$

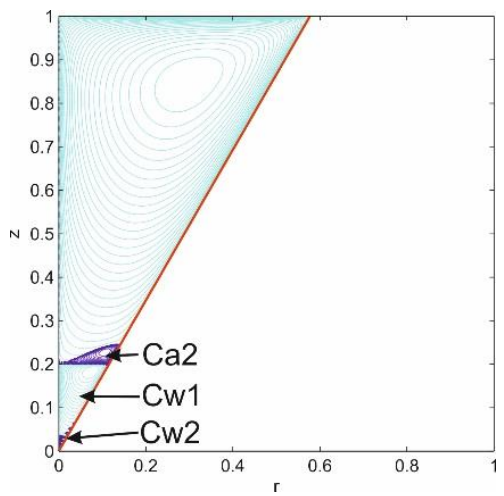

(b) $H_{\mathrm{w}}=0.201$

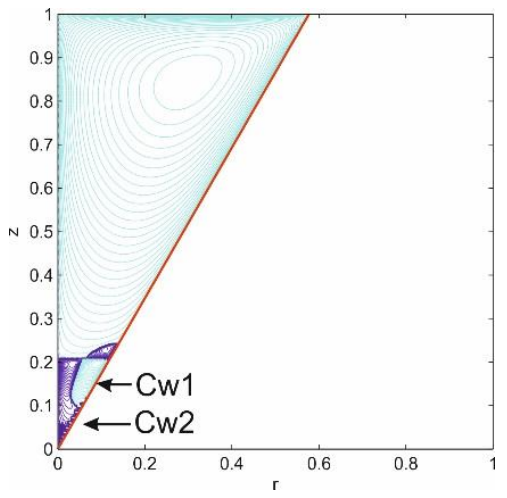

(e) $H_{\mathrm{w}}=0.208$

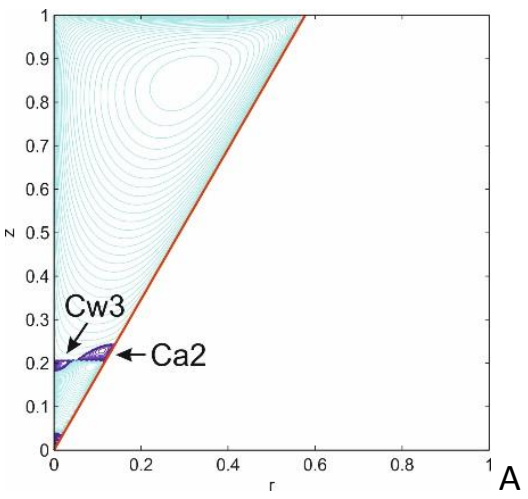

(c) $H_{\mathrm{w}}=0.205$

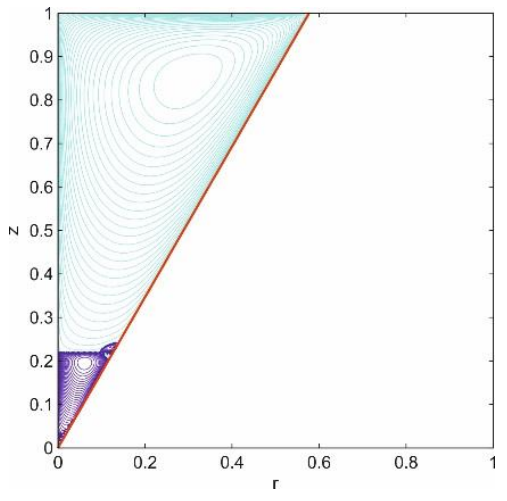

(f) $H_{\mathrm{w}}=0.22$

FIG. 2. (color online) Patterns of the meridional motion for small $H_{\mathrm{w}}$ at $\beta=30^{\circ}$. 
It is known that a single-fluid flow (e.g., with no water) has a cascade of eddies with alternating circulation directions [13]. The dimensions and strengths of eddies tend to zero as the cone tip is approached. Our numerical technique resolves only some of the cascade eddies.

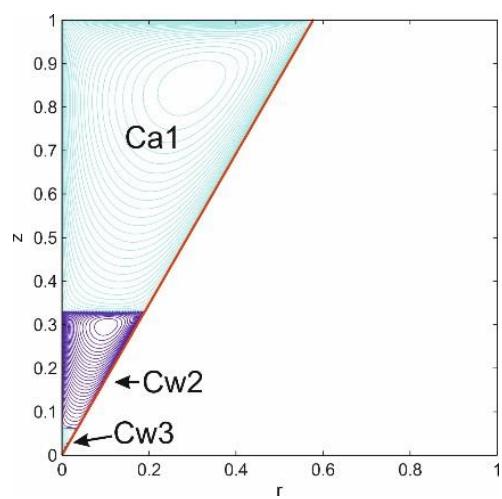

(a) $H_{\mathrm{w}}=0.33$

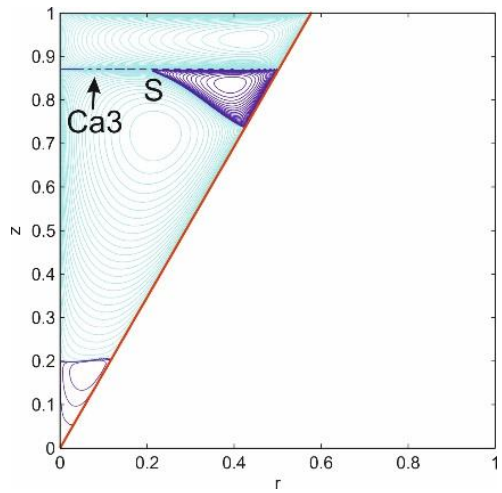

(d) $H_{\mathrm{w}}=0.87$

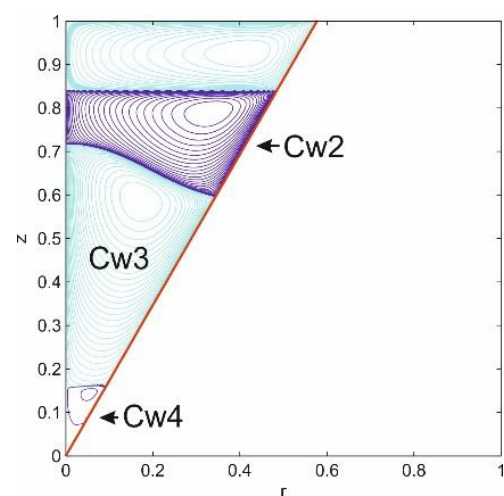

(b) $H_{\mathrm{w}}=0.84$

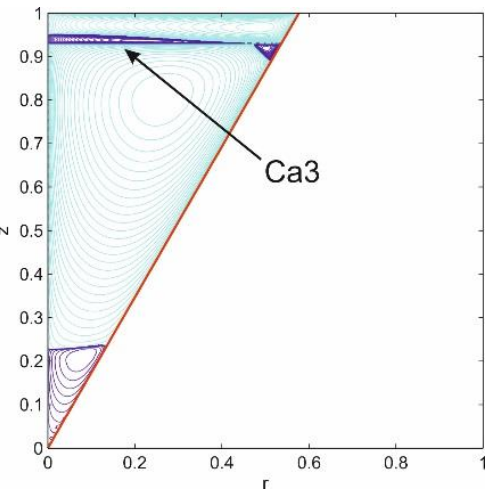

(e) $H_{\mathrm{w}}=0.93$

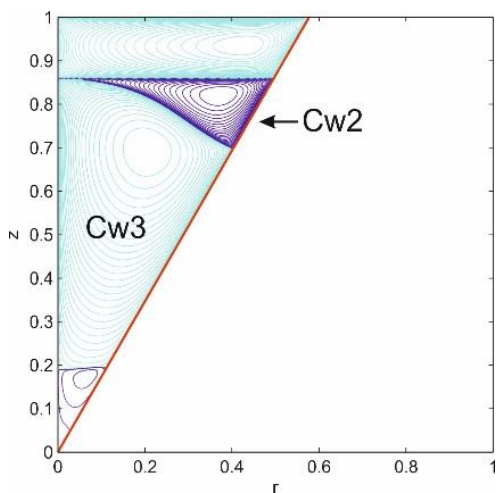

(c) $H_{\mathrm{w}}=0.86$

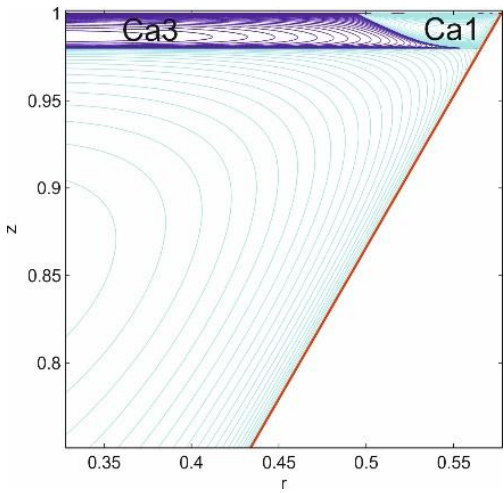

(f) $H_{\mathrm{w}}=0.98$

FIG. 3. (color online) Patterns of the meridional motion for large $H_{\mathrm{w}}$ at $\beta=30^{\circ}$. The effect of swirl results in expansion on of cell $\mathrm{Cw} 3$ and emergence of $\mathrm{Ca} 3$, as $H_{\mathrm{w}}$ increases.

Figure 2(a) shows two eddies in the air, $\mathrm{Ca} 1$ and $\mathrm{Ca} 2$, and two eddies in the water, $\mathrm{Cw} 1$ and $\mathrm{Cw} 2$, at $H_{\mathrm{w}}=0.1$; since $\mathrm{Cw} 1$ and $\mathrm{Cw} 2$ are very small in Fig. 2(a) they are indicated in Figure 2(b). 'C', 'a' and 'w' are abbreviations for 'cell', 'air' and 'water', respectively. Contours, corresponding to the clockwise (anticlockwise) circulation are depicted hereafter by the light (dark) curves which are blue online.

As $H_{\mathrm{w}}$ increases, region $\mathrm{Ca} 2$ shrinks and its upper boundary touches the interface at the axis as Fig. 2(b) illustrates at $H_{\mathrm{w}}=0.201$. Next, Ca2 separates from the axis thus transforming from a bubble-like into a ring-like shape. At the same value of $H_{\mathrm{w}}$, the clockwise air circulation in 
Ca1 induces a new cell, Cw3, in the water as Fig. 2(c) illustrates at $H_{\mathrm{w}}=0.205$. We denote this change in the flow topology as the first bifurcation, $\mathrm{b}_{1}$.

As $H_{\mathrm{w}}$ further increases, region Cw3 expands downward, as Fig. 2(d) shows at $H_{\mathrm{w}}=0.207$ and merges with region Cw2 as Fig. 2(e) reveals at $H_{\mathrm{w}}=0.208$. Region Cw1 becomes separated from the axis thus transforming from a bubble-like (Fig. 2(d)) into a ring-like (Fig. 2(e)) shape.

We mark this change in the flow topology as $\mathrm{b}_{2}$ and denote the merged region again as $\mathrm{Cw} 2$ in Fig. 2(e). For larger $H_{\mathrm{w}}$, regions $\mathrm{Ca} 2$ and $\mathrm{Cw} 1$ shrink to the interface-sidewall intersection, as Fig. 2(f) illustrates at $H_{\mathrm{w}}=0.22$.

Note that in addition to the cascade of eddies near the cone tip, a cascade of eddies also exists near the interface-sidewall intersection [18]. $\mathrm{Ca} 2$ and $\mathrm{Cw} 1$ can be considered as the outmost eddies of this additional cascade. However as $H_{\mathrm{w}}$ increase, $\mathrm{Ca} 2$ and $\mathrm{Cw} 1$ become so small that our grid cannot resolve them in Fig. 3 in contrast to Fig. 2(f).

Figure 3(a) depicts the flow pattern at $H_{\mathrm{w}}=0.33$, where three eddies are observed in the water: $\mathrm{Cw} 2, \mathrm{Cw} 3$, and $\mathrm{Cw} 4$; $\mathrm{Cw} 4$ is well seen in Fig. 3(b). They are driven by the meridional circulation of air in Ca1 while the direct effect of the centrifugal force is comparatively small. The solid curve in Fig. 4 depicts the dependence on $H_{\mathrm{w}}$ of the maximal swirl velocity at the interface, $v_{\mathrm{im}}$, and reveals the strong decay of $\mathrm{v}_{\mathrm{im}}$ as $H_{\mathrm{w}}$ decreases at $\beta=30^{\circ}$. This explains why the direct effect of swirl on the water motion is negligible at $H_{\mathrm{w}}=0.33$.

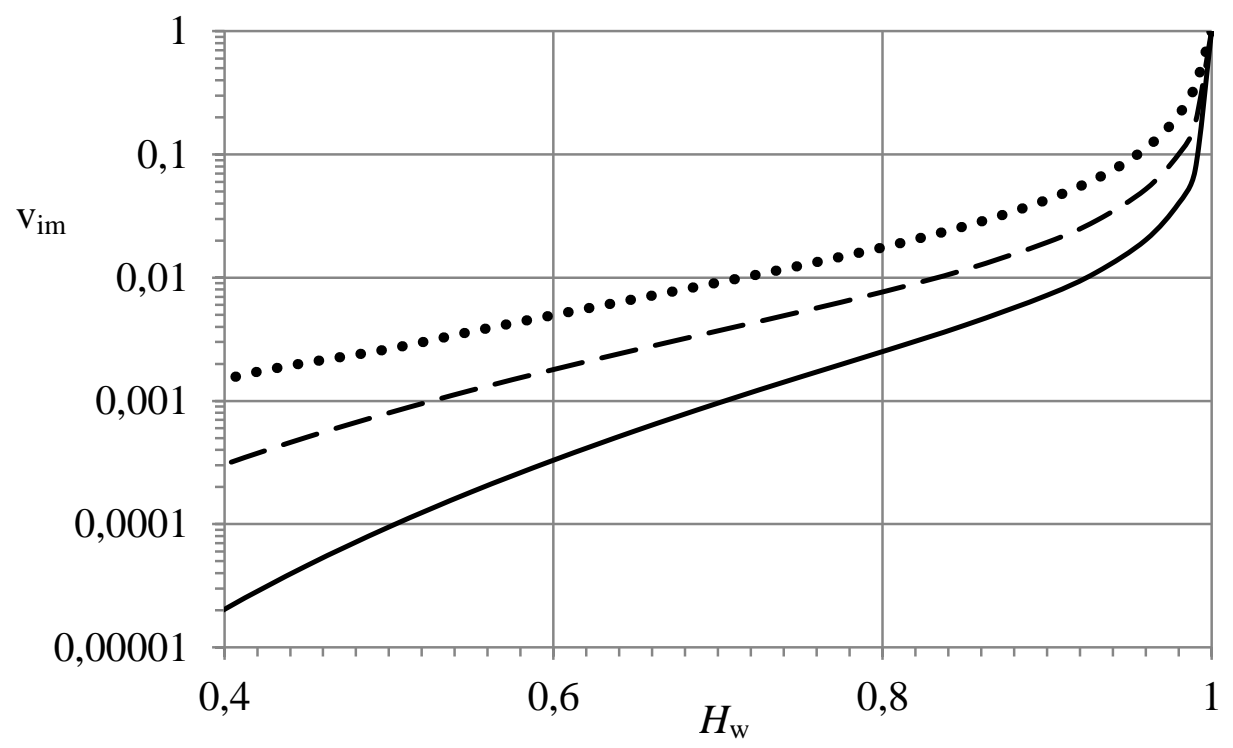

FIG. 4. The maximal swirl velocity at the interface versus the water height at $\beta=30^{\circ}$ (solid curve), $45^{\circ}$ (dashed curve) and $60^{\circ}$ (dots). 
The effect of swirl becomes significant as $H_{\mathrm{w}}$ increases. The centrifugal force pushes the fluid to the periphery near the top of a cell and thus enhances (suppresses) the clockwise (anticlockwise) circulation. Figures 3(a) and 3(b) illustrate this: region Cw3 (Cw2) expands (shrinks) as $H_{\mathrm{w}}$ increases.

Cw3 mostly expands near the axis (because water moves up there) and reaches the interface at $H_{\mathrm{w}}=0.86$ as Fig. 3(c) illustrates. For large $H_{\mathrm{w}}, \mathrm{Cw} 2$ separates from the axis. The water flow in $\mathrm{Cw} 3$ drives the adjacent air away from axis and thus generates cell Ca3. We mark this change in the flow topology as $\mathrm{b}_{3}$. Regions $\mathrm{Ca} 1, \mathrm{Ca} 3, \mathrm{Cw} 2$ and $\mathrm{Cw} 3$ meet at a saddle point $\mathrm{S}$ located at the interface as shown in Fig. 3(d).

$\mathrm{Ca} 3$ is a very thin layer hardly visible in Fig. 3(d) at $H_{\mathrm{w}}=0.87$ and well observed in Fig. 3(e) at $H_{\mathrm{w}}=0.93$. Its z-extent is maximal at the axis and diminishes to zero at $\mathrm{S}$. As $H_{\mathrm{w}}$ increases, $\mathrm{Ca} 3$ touches the lid and Ca1 becomes separated from the axis as Fig. 3(f) illustrates at $H_{\mathrm{w}}=0.98$. We mark this change in the flow topology as $b_{4}$.

Next, the saddle $\mathrm{S}$ reaches the sidewall at $H_{\mathrm{w}}$ around 0.98 and cell Ca1 becomes separated from the interface. We mark this change in the flow topology as $b_{5}$. Finally, Ca1 shrinks to the lid-sidewall intersection as $H_{\mathrm{w}} \rightarrow 1$.

\section{Bifurcations at $\beta=4^{\circ}$}

The bifurcation sequence at $\beta=45^{\circ}$ is similar to that at $\beta=30^{\circ}$, but the topological changes occur for $\beta=45^{\circ}$ at smaller $H_{\mathrm{w}}$ than for $\beta=30^{\circ}$. For example, the flow pattern shown in Fig. 5(a) is similar to that in Fig. 2(b) and the pattern shown in Fig. 5(b) is similar to that in Fig. 2(f). The bifurcations $b_{1}$ and $b_{2}$, occurring in the $H_{\mathrm{w}}$ range around 0.2 at $\beta=30^{\circ}$, also occur at $\beta=45^{\circ}$, but in the $H_{\mathrm{w}}$ range around 0.075 .

Figure 5(c) depicts the clockwise circulation in the water (region $\mathrm{Cw} 3$ ). As $H_{\mathrm{w}}$ increases, Cw3 expands and touches the interface at $H_{\mathrm{w}}=0.793$ (compare with $H_{\mathrm{w}}=0.86$ at which bifurcation $b_{3}$ occurs for $\beta=30^{\circ}$ ). Figures $5(\mathrm{~d})$ and $5(\mathrm{e})$ depict the flow patterns before and after bifurcation $b_{3}$. Similarly, bifurcations $b_{4}$ and $b_{5-0 c c u r}$ at smaller $H_{\mathrm{w}}$ at $\beta=45^{\circ}$ than those at $\beta=$ $30^{\circ}$. Figure 5(f) depicts the flow pattern after $\mathrm{b}_{4}$.

The dashed curve in Fig. 4 illustrates that the swirl decays weaker for $\beta=45^{\circ}$ than that for $\beta$ $=30^{\circ}$ as the distance of the interface from the rotating disk increases. The dotted curve in in Fig. 
4 corresponds to $\beta=60^{\circ}$. This case, where the swirl decays weaker than that for $\beta=45^{\circ}$, is discussed in more details below.

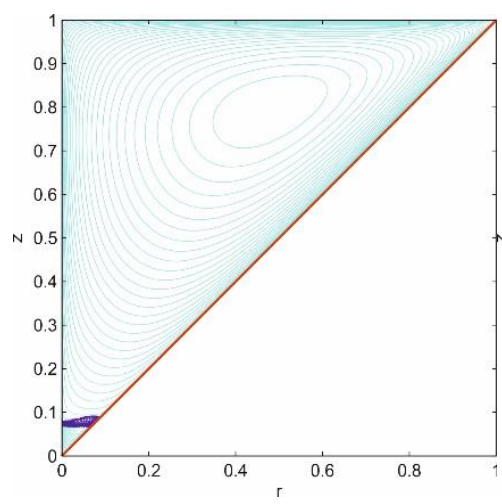

(a) $H_{\mathrm{w}}=0.07$

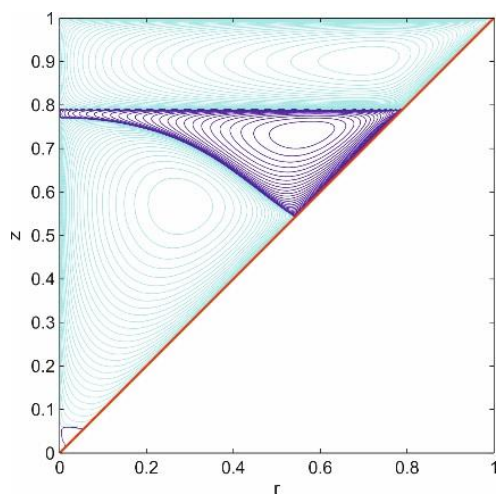

(d) $H_{\mathrm{w}}=0.79$

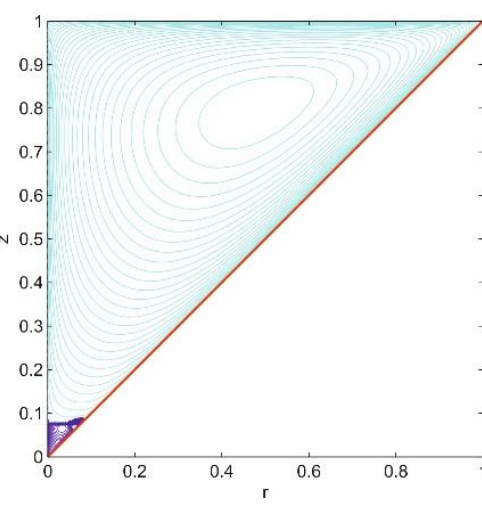

(b) $H_{\mathrm{w}}=0.08$

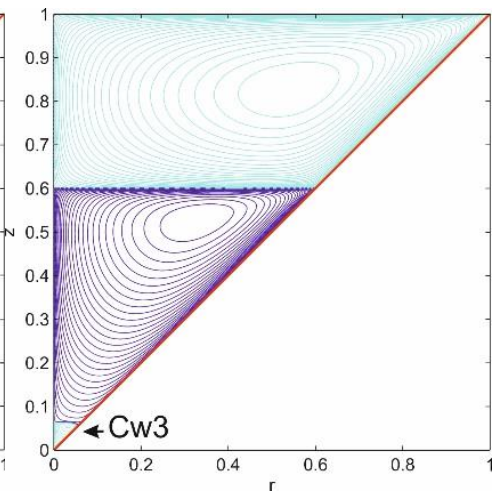

(c) $H_{\mathrm{w}}=0.6$

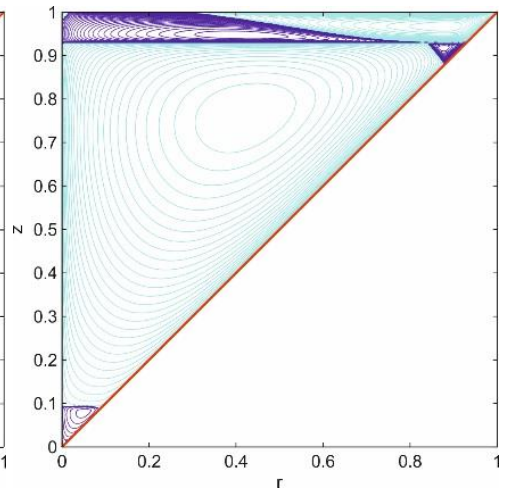

(c) $H_{\mathrm{w}}=0.93$

FIG. 5. (color online) Flow patterns at $\beta=45^{\circ}$.

\section{Bifurcations at $\beta=60^{\circ}$}

In contrast to the cases where $\beta=30^{\circ}$ and $45^{\circ}$, the air flow is one-cellular for $\beta=60^{\circ}$ even at $H_{\mathrm{w}}=0.02$ as Fig. 6(a) illustrates. Another difference is that no eddy is observed near the interface-sidewall intersection. This agrees with the theory [18] stating that if a flow is driven from the air side and $\beta>56.55^{\circ}$, no Moffatt eddy occurs near the interface-sidewall intersection. On the other hand according to the theory [18], there is a cascade of eddies in the water flow near the cone tip at $\beta=60^{\circ}$. The numerical grid, we apply, can resolve none of these eddies at $H_{\mathrm{w}}=$ 
0.02 in Fig. 6(a). However as $H_{\mathrm{w}}$ increases, some of the cascade eddies become visible as Fig. 6(c) and 6(d) clearly show. Figure 6(b) depicts a pattern close to the eddy Cw3 resolution boundary.

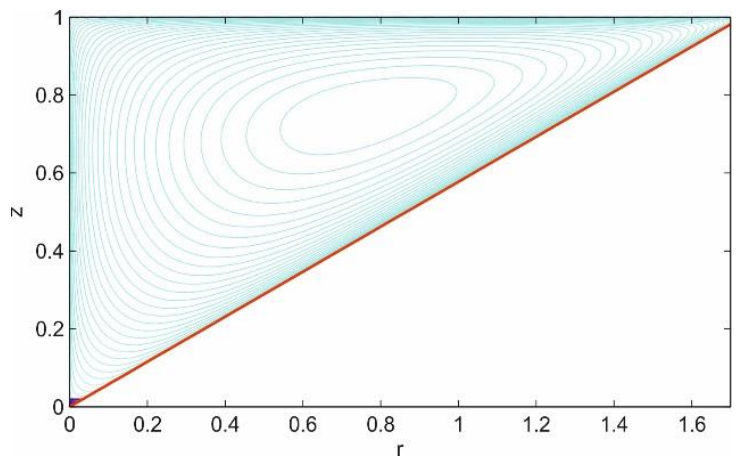

a) $H_{\mathrm{w}}=0.02$

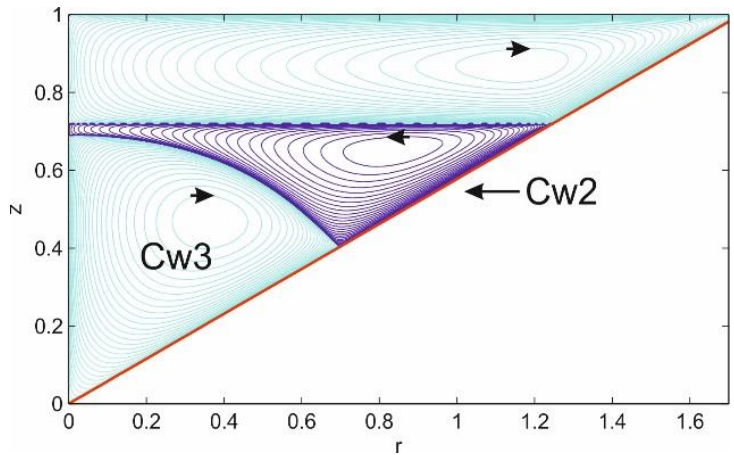

(c) $H_{\mathrm{w}}=0.72$

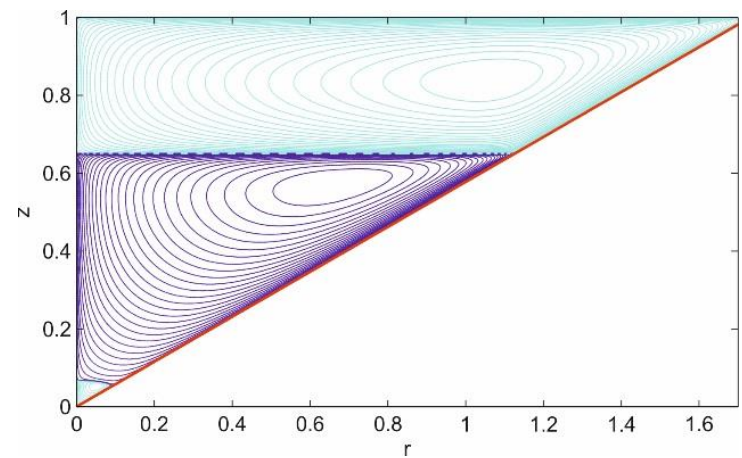

(b) $H_{\mathrm{w}}=0.65$

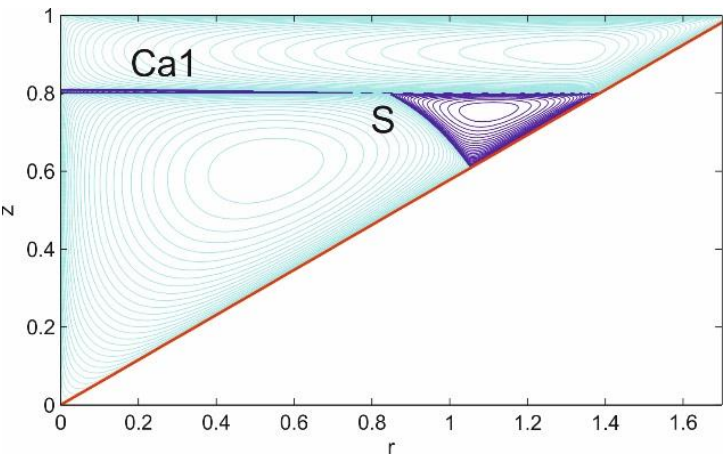

(d) $H_{\mathrm{w}}=0.8$

FIG. 6. (color online) Development of counter-circulation in water, (a)-(b), and in air, (c)-(d), at $\beta=60^{\circ}$.

Figure 7 helps to clarify this issue by presenting the dependence on $z$ of velocity $w$ on the axis in the water flow for a few values of $H_{\mathrm{w}}$. The z-ranges where $w>0(w<0)$ correspond to the clockwise (anticlockwise) circulation, e.g., as depicted in region $\mathrm{Cw} 3(\mathrm{Cw} 2)$ in Figure 6(c). As $H_{\mathrm{w}}$ decrease, the $w>0$ range shrinks and is not observed for $H_{\mathrm{w}}=0.64$ (interpolation predicts that the $w>0$ range disappears at $\left.H_{\mathrm{w}}=0.647\right)$.

Bifurcations $b_{3}, b_{4}$, and $b_{5}$ at $\beta=60^{\circ}$ are similar to those at $\beta=30^{\circ}$ and $45^{\circ}$, but occur at smaller $H_{\mathrm{w}}$. For example, the saddle $\mathrm{S}$, shown at $H_{\mathrm{w}}=0.8$ in Fig. 6(d), separates from the axis at $H_{\mathrm{w}}=0.729$ and reaches the sidewall at $H_{\mathrm{w}}=0.942$. Region Cal separates from the axis at $H_{\mathrm{w}}=$ 0.981 . 
To explore in what range of the Reynolds number, $R e$, the flow topology remains the same as that for the creeping flows, we below explore how the flow pattern changes as $R e$ increases.

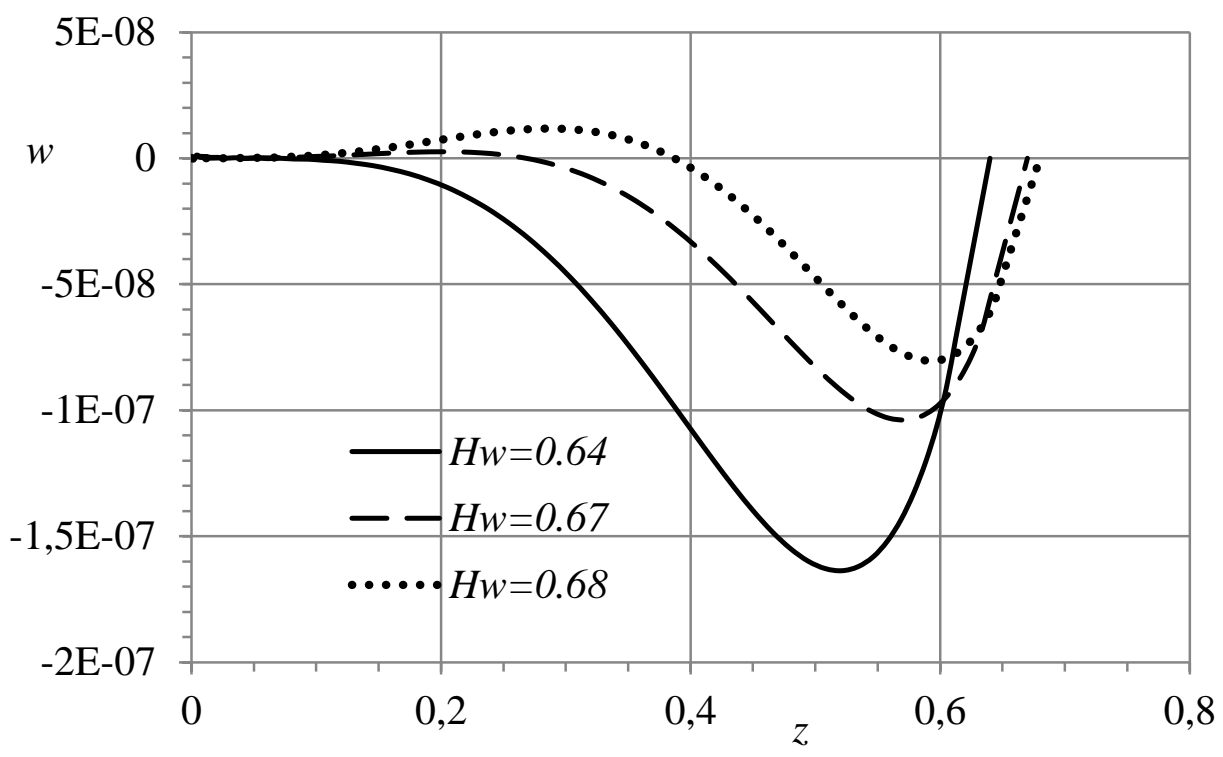

FIG. 7. (color online) Profiles of velocity at the axis in water show the counter-circulation (w > $0)$ emergence as $H_{\mathrm{w}}$ increases at $\beta=60^{\circ}$.

\section{The effect of intensifying disk rotation}

Now we come back from the reduced equations ( $\$ 2.4)$ to the full equations $(\S 2.2$ and $\S 2.3)$ and use the numerical techniques for the nonlinear problem ( 33$)$. For $R e>0$, the interface becomes deformed. The deformation depends on the Froude number, $F r=\omega^{2} R / g=a R e^{2}$, and the Weber number, $W e=\rho_{\mathrm{w}} \omega^{2} R^{3} / \sigma=b R e^{2} ; a=v_{\mathrm{w}}{ }^{2} /\left(\mathrm{gR}^{3}\right)$ and $b=\rho_{\mathrm{w}} v_{\mathrm{w}}{ }^{2} /(R \sigma)$. We fix $a$ and $b$ while increase $R e$. The bioreactor radius, $R$, is around $0.02 \mathrm{~m} \mathrm{[4];} \mathrm{this} \mathrm{yields} a=1.25 \times 10^{-8}$, and $b=$ $7 \times 10^{-7}$ at $R=0.02 \mathrm{~m}$. For the range $\operatorname{Re}$ considered here, $\mathrm{Fr}, \mathrm{We}$, and interface deformation are small.

To characterize the strength of air flow, we introduce the Reynolds number based on the air viscosity, $R e_{\mathrm{a}}=\omega h^{2} / \mathrm{v}_{\mathrm{a}}=R e / \mathrm{v}_{\mathrm{n}}$, because the rotating top disk directly (indirectly) drives the air (water) flow; we take the air-to water kinematic viscosity ratio being $v_{n}=15$. From the top disk down to the interface, the swirl velocity significantly drops. Therefore, to characterize the 
strength of water flow, we introduce the Reynolds number, $R e_{\mathrm{w}}$, based on the maximal swirl velocity at the interface, $\mathrm{v}_{\mathrm{im}} ; R e_{\mathrm{w}}=\mathrm{v}_{\mathrm{im}} R e$.

We increase $R e$ at the fixed values of $\beta=60^{\circ}$ and $H_{\mathrm{w}}=0.8$. Our goal is to find at what $R e$ the flow topology becomes different from that presented in Fig. 6(d). As Re increases, the saddle $\mathrm{S}$, shown in Fig. 6(d), shifts toward the axis, as Fig. 8(a) depicts. The radial coordinate, $r_{\mathrm{s}}$, of $\mathrm{S}$ decreases as Table 1 indicates. The saddle $S$ reaches the axis at $R e$ around 10000 and the flow pattern becomes as Fig. 8(b) depicts.

Comparison of Figures 8(a) and 8(b) shows that the clockwise circulations in air (Ca1) and in water $(\mathrm{Cw} 3)$ become separated by the anticlockwise circulation in water $(\mathrm{Cw} 2)$. The z-extension of $\mathrm{Cw} 2$ shrinks as $R e$ increases. This feature is the same as that observed in the water-spout cylindrical flow [25].

\begin{tabular}{|r|r|r|r|}
\hline \multicolumn{1}{|l|}{$R$ R } & \multicolumn{1}{l|}{$R e_{\mathrm{a}}$} & \multicolumn{1}{l|}{$\boldsymbol{r}_{\mathrm{s}}$} \\
\hline 0.1 & 0.0067 & 0.00177 & 0.607 \\
\hline 2000 & 133 & 34.4 & 0.59 \\
\hline 4000 & 267 & 64.7 & 0.553 \\
\hline 6000 & 400 & 90.6 & 0.499 \\
\hline 8000 & 533 & 112 & 0.346 \\
\hline 10000 & 667 & 135 & 0 \\
\hline 20000 & 1333 & 320 & 0 \\
\hline
\end{tabular}

Table 1 Dependence on $R e$ of the Reynolds number for air $\left(R e_{\mathrm{a}}\right)$ and water $\left(R e_{\mathrm{w}}\right)$ flows and of the radial coordinate of saddle point $\mathrm{S}$ (se Fig. $6(\mathrm{~d})$ ) at $H_{\mathrm{w}}=0.8$ and $\beta=60^{\circ}$. 


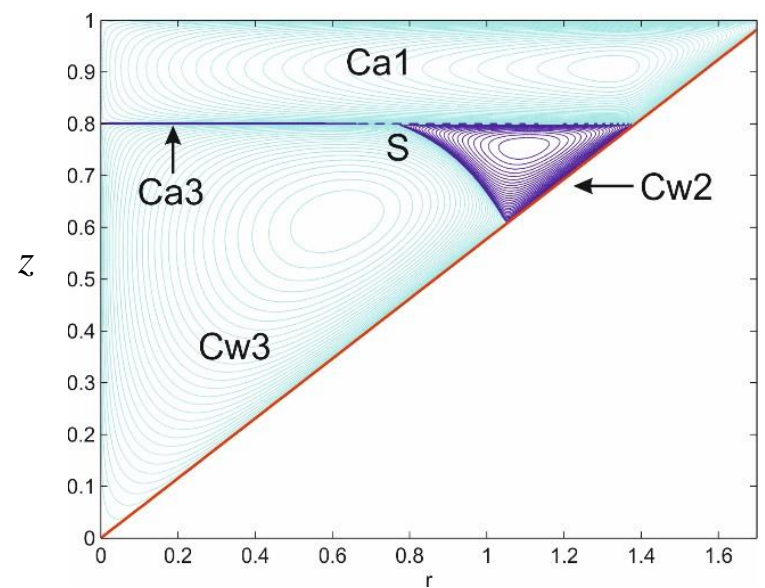

(a) $\operatorname{Re}=4000$

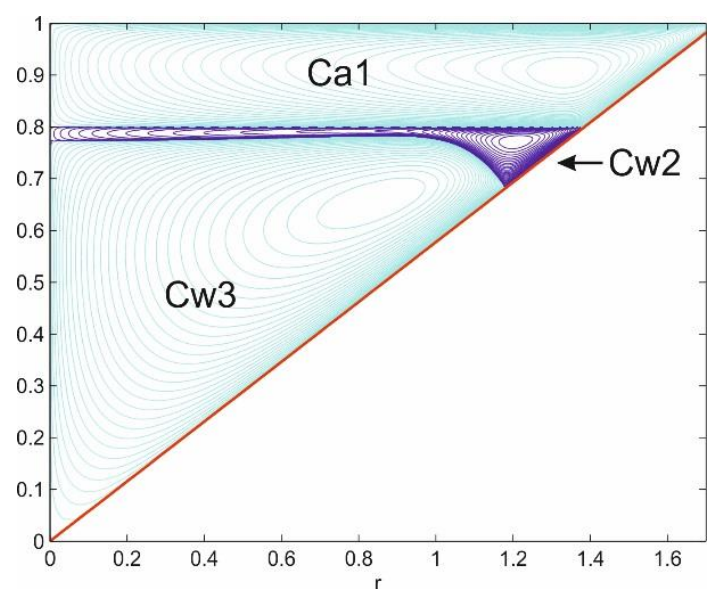

(b) $R e=20000$

FIG. 8. (color online) Transformation of the flow pattern as $R e$ increases at $H_{\mathrm{w}}=0.8$ and $\beta=$ $60^{\circ}$.

\section{Conclusions}

This paper reveals and explains topological transformations in a slow steady axisymmetric airwater flow, driven by the rotating top disk, in a vertical conical container. There are five major flow bifurcations as the water height $H_{\mathrm{w}}$ varies at the cone half-angle $\beta=30^{\circ}$ (Sect. 4). Most of the bifurcations occur at the interface. For small $H_{\mathrm{w}}$, the direct effect of swirl is negligible for the water flow where a cascade of eddies exists near the cone tip. As $H_{\mathrm{w}}$ increases, the swirl effect becomes significant and causes the expansion of the outmost cascade eddy with the clockwise circulation (Fig. 2). As this eddy reaches the interface, it reverses the adjacent air flow developing a thin layer with the anticlockwise circulation (Fig. 3). These expansions and reversals occur for smaller $H_{\mathrm{w}}$ at $\beta=45^{\circ}$ (Sect. 5, Fig. 5) and $\beta=60^{\circ}$ (Sect. 6, Fig. 6) than those at $\beta=30^{\circ}$. As the disk rotation intensifies, the flow topology remains invariant until the Reynolds number $R e$ exceeds a value around $10^{4}$ (Sect. 7, Table 1). For larger $R e$, the bulk circulations of both air and water flows are clockwise, separated by a thin layer of anticlockwise circulation adjacent to the interface from the water side (Fig. 8). The physical reasons for the flow transformations are provided. The results are of fundamental interest and can be relevant for aerial bioreactors. 
[1] Shtern, V.: Counterflows. Cambridge University Press (2012).

[2] Herrada, M. A., Shtern, V. N.: Patterns of a creeping water-spout flow. J. Fluid Mech. 744, 65 (2014).

[3] Balci, A., Brøns, M., Herrada, M. A., Shtern, V.N.: Vortex breakdown in a truncated conical bioreactor. Fluid Dyn. Res. 47, 065503 (2015).

[4] Liow, K. Y. S., Tan, B. T., Thouas, G., Thompson, M. C.: CFD modeling of the steady-state momentum and oxygen transport in a bioreactor that is driven by a rotating disk. Modern Physics Letters B 23, 121 (2009).

[5] Ramazanov, Yu. A., Kislykh, V.I., Kosyuk, I.P., Bakuleva, N.V., Shchurikhina, V.V.: Industrial production of vaccines using embryonic cells in gas-vortex gradient-less bioreactors. In book "New Aspects of Biotechnology and Medicine”, edited by A. M. Egorov, ISBN: 1-60021-465-7, pp. 87-91 (2007).

[6] Shtern, V. N., Torregrosa, M. M., Herrada, M. A.: Effect of swirl decay on vortex breakdown in a confined steady axisymmetric flow. Phys. Fluids 24, 043601 (2012).

[7] Bödewadt, U. T.: Die Drehströmung über festem Grund. Z. Angew. Math. Mech. 20, 241 (1940).

[8] Ackerberg, R. C.: The viscous incompressible flow inside a cone. J. Fluid Mech. 21,47 (1965).

[9] Schultz, V., Gorbach, G., Piesche, M.: Modeling fluid behavior and droplet interactions during liquid-liquid separation in hydrocyclones. Chem. Eng. Sci., 64, 3935 (2009).

[10] Ghodraty, M., Kuang, S. B., Yui, A. B., Vince, A., Barnett, G. D., Barnet, P. J.: CFD study of multiphase flowin classifying cyclone: Effect of cone geometry. Ninth Int. Conf. on CFD 
in the Mineral and Process Industries CSIRO, Melbourne, Australia 10-12 December 2012.

[11] Secchiaroli, A., Ricci, R., Montelpare, S. D’Alessandro, V.: Numerical simulation of turbulent flow in a Ranque-Hilsch vortex-tube. Int. J. of Heat and Mass Transfer 52, 5496 (2009).

[12] Moffatt, H. K.: Viscous and resistive eddies near a sharp corner. J. Fluid Mech. 18, 1 (1964).

[13] Wakiya, S.: Axisymmetric flow of a viscous fluid near the vertex of a body. J. Fluid Mech. 78, 737 (1976).

[14] Liu, C. H., Joseph, D. D.: Stokes flow in conical trenches, SIAM J. Appl. Math. 34, 286 (1978).

[15] Weidman, P. D., Calmidi, V.: Instantaneous Stokes flow in a conical apex aligned with gravity and bounded by a stress-free surface. SIAM J. Appl. Maths. 59, 1520 (1999).

[16] Malyuga, V. S.: Viscous eddies in a circular cone. J. Fluid Mech. 522, 101 (2005).

[17] Shankar, P. N.: Moffatt eddies in the cone. J. Fluid Mech. 539, 113 (2005).

[18] Shtern, V.: Moffatt eddies at an interface. Theor. Comput. Fluid Dyn. 28, 651 (2014 ).

[19] Brady, P. T., Herrmann, M., Lopez, J. M.: Two-fluid confined flow in a cylinder driven by a rotating end wall", Phys. Rev. E 85, 016308 (2012).

[20] Arnold, V.I.: Sur la gćometrie diffćrentielle des groupe de Lie de dimension infinie et ses applications a l'hydrodynamique des fluides parfaits. Ann. Inst Fourier 16, 319 (1966).

[21] Arnold, V.I., Khesin, B. A.: Topological methods in hydrodynamics. Applied Math. Sci. 125, Springer-Verlag, N.Y (1998).

[22] Moffatt, H. K., Tsinober, A. (editors): Topological Fluid Mechanics. Proc. of IUTAM Symp. Cambridge, UK (1989). 
[23] Moffatt, H. K., Bajer, K., Kimura, Y. (editors): IUTAM Symposium on Topological Fluid Dynamics: Theory and Applications. Procedia IUTAM 7, 1-260. Elsevier (2013).

[24] Hills, C. P. Eddies induced in cylindrical containers by a rotating end wall. Phys. Fluids 13, 2279 (2001).

[25] Herrada, M. A., Shtern, V. N., Lopez-Herrera, J. M.: Vortex breakdown in a water-spout flow. Phys. Fluids 25, 093604 (2013). 